Assurances et gestion des risques

Insurance and Risk Management

ASSURANCES

\title{
Construire un générateur de scénarios économiques risque neutre
}

\section{Kamal Armel et Frédéric Planchet}

Volume 85, numéro 1-2, juin 2018

URI : https://id.erudit.org/iderudit/1051316ar

DOI : https://doi.org/10.7202/1051316ar

Aller au sommaire du numéro

Éditeur(s)

Faculté des sciences de l'administration, Université Laval

ISSN

1705-7299 (imprimé)

2371-4913 (numérique)

Découvrir la revue

Citer ce document

Armel, K. \& Planchet, F. (2018). Construire un générateur de scénarios

économiques risque neutre. Assurances et gestion des risques / Insurance and

Risk Management, 85(1-2), 57-94. https://doi.org/10.7202/1051316ar

Tous droits réservés $\odot$ Faculté des sciences de l'administration, Université Laval, 2018
Ce document est protégé par la loi sur le droit d'auteur. L'utilisation des services d'Érudit (y compris la reproduction) est assujettie à sa politique d'utilisation que vous pouvez consulter en ligne.

https://apropos.erudit.org/fr/usagers/politique-dutilisation/ 


\title{
CONSTRUIRE UN GÉNÉRATEUR DE SCÉNARIOS ÉCONOMIQUES RISQUE NEUTRE
}

\author{
Kamal ARMEL ${ }^{1}$, Frédéric PLANCHET ${ }^{2}$
}

Ce document est rattaché à l'article Armel et Planchet [2018]: «Comment construire un générateur de scenarios économiques risque neutre destiné à l'évaluation du best-estimate des contrats d'épargne?». Il présente une démarche de construction d'un générateur de scénarios économiques risque neutre dans le cadre d'un environnement économique caractérisé par des taux négatifs.

\section{TABLE DES MATIÈRES}

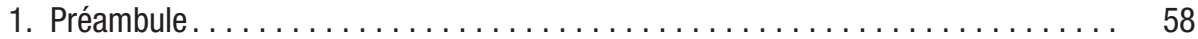

2. GSE risque neutre: modélisation, calibrage et simulation............ 59

2.1 Modèles de taux . . . . . . . . . . . . . . . . . . . . . . . . 59

2.1.1 Modèles de Black........................... 61

2.1.2 Modèle Hull \& White . . . . . . . . . . . . . . . . . . . . 65

2.1.3 Modèle gaussien linéaire à deux facteurs $(\mathrm{G} 2++) \ldots \ldots \ldots \ldots \ldots, 72$

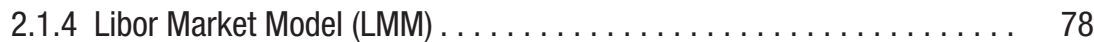

2.2 Modèle actions et immobilier . . . . . . . . . . . . . . . . . . . 86

2.2.1 La dynamique du modèle Black \& Scholes . . . . . . . . . . . . 86

2.2.2 Solution de l'équation différentielle.................. 87

2.2.3 Valorisation d'une option d'achat européenne .............. 88

2.2.4 Calibrage du modèle Black et Scholes sur un indice actions . ........ 89

2.2.5 Calibrage du modèle Black et Scholes sur un indice immobilier . . . . . 89

2.3 Modélisation de la structure de dépendance . . . . . . . . . . . . . . 89

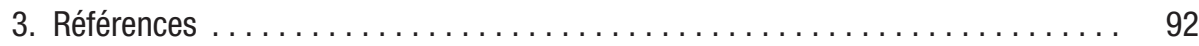




\section{Préambule}

Le processus de génération de scénarios économiques peut être synthétisé en trois étapes (Laurent et al. [2016], Planchet et al. [2009], Baldvinsdóttir et Palmborg [2011], Ballotta [2004], Armel et al. [2011]...):

1. L'environnement de modélisation: il s'agit de choisir les variables économiques à modéliser dans un univers probabilisé. Classiquement, la mesure retenue est une probabilité risque neutre. En effet, la génération de scénarios économiques Market Consistent sous une probabilité historique rencontre systématiquement la problématique de la quantification des primes de risque. S'en affranchir est pratique. Le passage à une probabilité risque neutre ouvre cette possibilité. Une synthèse de l'approche par les déflateurs en probabilité historique est décrite dans Caja et Planchet [2010], Cheng et Planchet [2018] et un exemple de mise en ouvre simplifié est proposé dans Dastarac et Sauveplane [2010].

2. Les modèles: il s'agit de construire les modèles mathématiques des variables d'intérêt. Cela consiste à choisir les modèles qui représentent la dynamique marginale de ces variables et la structure de dépendance.

3. Les paramètres et le calibrage: il s'agit de choisir les produits financiers dérivés pour les calibrages, les données, les méthodes d'estimation statistique des paramètres des modèles et des méthodes de validation. Armel et al. [2011] a montré d'ailleurs que le choix de la copule destinée à représenter la structure de dépendance d'un GSE dépend de la distance retenue pour mesurer les écarts entre une copule empirique et une copule paramétrique.

Ces étapes peuvent se schématiser comme présenté dans la Figure 1.

\section{FIGURE 1 Illustration schématique des choix de modélisation et de calibrage d'un GSE}
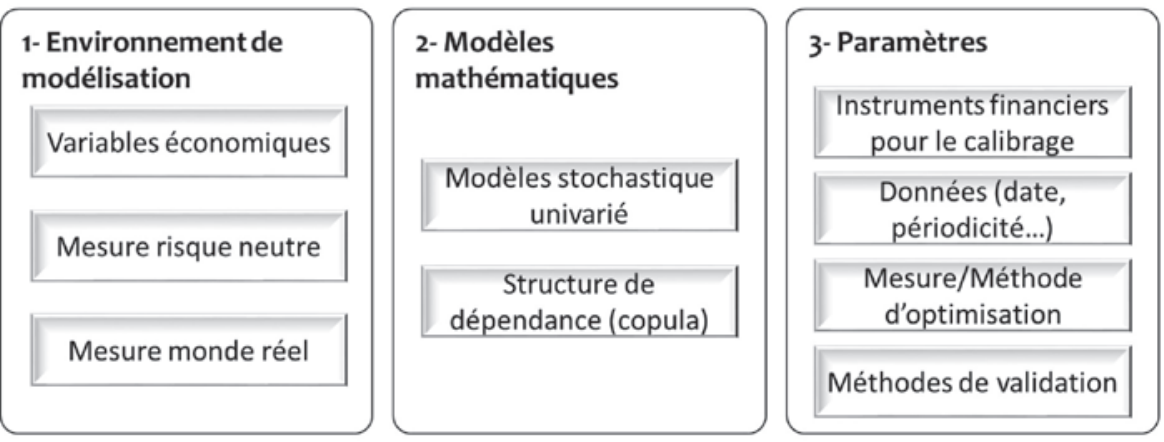
Ce document présente une démarche de construction d'un GSE dont les caractéristiques sont les suivantes:

- La mesure de probabilité est une probabilité risque neutre;

- Les variables retenues sont les taux d'intérêt sans risque et des actifs risqués tels que les actions, les investissements en immobilier et les investissements en infrastructure. Dans la suite seuls deux actifs risqués sont présentés: actions et immobilier. Le risque de crédit n'est pas abordé ici. La démarche proposée dans ce papier peut intégrer une infinité d'actifs risqués de type actions.

- Trois modèles de taux sont proposés :

- Un modèle mono-factoriel: Hull \& White calibré sur des caps et sur des swaptions;

- Un modèle à deux facteurs: modèle gaussien G2++ calibré sur des caps et sur des swaptions;

- Un modèle de marché: Libor Market Model (LMM) calibré sur des swaptions.

- Les prix des actions et des investissements en immobilier sont supposés suivre un mouvement brownien de type Black et Scholes sous la probabilité risque neutre;

- La structure de dépendance retenue est la corrélation: nous nous plaçons donc dans un cadre gaussien.

La section suivante présente le cadre théorique de construction d'un générateur de scénarios économiques risque neutre. Elle se compose de trois sous-sections présentant:

- Les modèles de taux;

- Les actifs risqués de type actions et investissements en immobilier;

- La structure de dépendance. 


\section{GSE risque neutre: modélisation, calibrage et simulation}

\subsection{Modèles de taux}

Les modèles de taux présentés dans la suite sont issus de deux familles largement étudiées dans la littérature financière:

- Les modèles dont la dynamique suit une loi normale comme le modèle de Hull-White (et plus généralement la famille des modèles $\mathrm{HJM}^{3}$ ) et le modèle à deux facteurs G2++;

- Les modèles de taux dont la dynamique suit une loi log-normale comme le modèle de marché LMM.

Le modèle Hull \& White peut être calibré pour reproduire la structure par terme des taux d'intérêt et la structure par terme des volatilités. Il permet également de valoriser les caps par une formule fermée et de générer des taux négatifs.

Brigo et Mercurio [2007] soulignent toutefois que la reproduction parfaite d'une structure par terme de la volatilité peut présenter certaines limites. En effet, les structures de volatilités implicites au modèle Hull \& White risquent d'être irréalistes dans la mesure où elles ne se conforment pas aux formes typiques du marché. Rebonato [2004] souligne également que la valorisation de caps par le modèle de Hull et White est moins précise que celle réalisée par le modèle LMM.

Par ailleurs, le modèle gaussien G2++ présente une flexibilité analytique qui facilite considérablement la valorisation des produits dérivés de taux. Le modèle propose en effet des formules fermées pour valoriser les caps/floors et les swaptions. Aussi, la présence de deux facteurs permet de mieux décrire la variabilité des taux du marché. Cela se traduit par un calibrage plus précis des swaptions européennes. Le modèle G2++ permet de générer des taux négatifs. Une description détaillée de ce modèle est présentée dans Brigo et Mercurio [2007].

Contrairement aux modèles Hull \& White et G2++ modélisant un processus caché sous-jacent aux prix observés (le taux court instantané), le modèle LMM décrit directement la dynamique des taux négociables sur un marché.

S'agissant d'un modèle log-normal, il génère uniquement des taux positifs qui sont dans certains cas assez importants. 
Le modèle LMM est largement utilisé par les praticiens. La principale raison réside dans sa capacité à reproduire des prix cohérents avec le marché pour les caps, les floors et les swaptions (Brigo et Mercurio [2007]). En effet, le modèle valorise :

- Les caps avec la formule de Black, qui est la formule standard utilisée sur le marché des caps;

- Les swaptions avec la formule de swaptions de Black, qui est également la formule standard utilisée sur le marché des swaptions.

Étant donné que les marchés des caps et des swaptions sont les deux principaux marchés d'options sur les taux d'intérêt, il est important qu'un modèle soit compatible avec ces formules de marché. Avant l'introduction des modèles LMM, aucune dynamique de taux d'intérêt n'était cohérente avec la formule de Black pour les caps et avec la formule de Black pour les swaptions.

Les formules de Black pour la valorisation de caps, de floors et de swaptions sont présentées dans la suite.

Notons cependant que les prix valorisés par le modèle LMM dépendent de la spécification des dynamiques de la volatilité instantanée et de la corrélation des taux forwards. Une étude détaillée de cette problématique est présentée dans Rebonato [2004] qui décrit et compare différents modèles de volatilité et de corrélation.

Les sections suivantes présentent les spécifications techniques des modèles de taux retenus ainsi que les méthodes de calibrage et de valorisation (zéro-coupon, caps/floors et swaptions). Nous nous sommes appuyés essentiellement sur Brigo et Mercurio [2007] pour développer et implémenter ces modèles.

\subsubsection{Modèles de Black}

L'utilisation de la formule de Black pour la valorisation des caps, des floors et des swaptions est une pratique courante du marché depuis des années, avant même l'élaboration d'un cadre cohérent avec l'absence d'opportunité d'arbitrage et avec la formule de Black (comme cela est le cas du modèle LMM). 
Outre les prix des obligations et les taux forwards observés, la formule de Black nécessite en argument la surface de volatilité observée sur le marché pour valoriser les caps, les floors et les swaptions. Il est noté néanmoins que les surfaces de volatilités ne résultent pas d'une mesure directe du prix mais d'une reconstruction par le fournisseur. Bloomberg utilise par exemple un modèle SABR ${ }^{4}$.

La dynamique du modèle de Black s'écrit: $d S_{\alpha, \beta}(\mathrm{t})=S_{\alpha, \beta}(t) \cdot \sigma_{\alpha, \beta} d W_{t}^{\alpha, \beta}$. Le sous-jacent, dont la volatilité est $\sigma_{\alpha, \beta}$, est noté $S_{\alpha, \beta}$ et $W_{t}^{\alpha, \beta}$ est un mouvement brownien. Les paramètres $(\alpha, \beta)$ représentent respectivement la date d'exercice et la maturité du sous-jacent.

\section{2.v1.1.1 Valorisation de caps et de floors}

La valorisation des caps et floors par le modèle de Black suppose que le taux forward suit la dynamique de Black présentée ci-dessus.

Notons $T=\left\{T_{\alpha}, \ldots, T_{\beta}\right\}$ l'ensemble des dates de paiement des caps/ floors et $T_{\alpha}$ le temps d'initialisation. Soit $\tau=\left\{\tau_{\alpha+1}, \ldots, \tau_{\beta}\right\}$ tel que $\tau_{i}$ représente la différence entre $T_{i-1}$ et $T_{i}$. Le prix à l'instant $t=0 \mathrm{du} c a p$ de prix d'exercice $K$ et de valeur nominale $N$ est donné par (Brigo et Mercurio [2007]):

$$
\operatorname{Cap}^{\text {Black }}\left(t=0, T, \tau, N, K, \sigma_{\alpha, \beta}\right)=N \sum_{i=\alpha+1}^{\beta} P\left(0, T_{i}\right) \tau_{i} B l\left(K, F\left(0, T_{i-1}, T_{i}\right), v_{i}, 1\right)
$$

Avec:

- $P\left(0, T_{i}\right)$ est le prix d'une obligation zéro-coupon observé en $t=0$ pour l'échéance $T_{i}$;

- $F\left(0, T_{i-1}, T_{i}\right)$ est le taux forward observé en $t=0$ pour la période entre $T_{i-1}$ et $T_{i}$;

- $B l(K, F, v, \omega)=F \omega \Phi\left(\omega d_{1}(K, F, v)\right)-K \omega \Phi\left(\omega d_{2}(K, F, v)\right)$;

- $\Phi$ la fonction de distribution gaussienne standard;

$-d_{1}(K, F, v)=\frac{\ln \left(\frac{F}{K}\right)+\frac{v^{2}}{2}}{v} ;$
- $d_{2}(K, F, v)=\frac{\ln \left(\frac{F}{K}\right)-\frac{v^{2}}{2}}{v} ;$ 
- $v_{i}=\sigma_{\alpha, \beta} \sqrt{T_{i-1}}$;

- $\sigma_{\alpha, \beta}$ est la volatilité (généralement extraite des cours du marché).

Le prix du floor est:

$$
\text { floor }^{\text {Black }}\left(t=0, T, \tau, N, K, \sigma_{\alpha, \beta}\right)=N \sum_{i=\alpha+1}^{\beta} P\left(0, T_{i}\right) \tau_{i} B l\left(K, F\left(0, T_{i-1}, T_{i}\right), v_{i},-1\right)
$$

Notons que le paramètre de volatilité implicite $\sigma_{\alpha, \beta}$. est totalement déterminé à partir du prix d'exercice, des prix de marché des obligations zéro-coupon sans risque et des produits dérivés (des caps ou des floors). En d'autres termes, étant donnés un prix d'exercice et une courbe de taux, la correspondance entre les prix des dérivés et les volatilités implicites est une bijection.

\subsubsection{Valorisation de swaptions}

La valorisation des caps et floors par le modèle de Black suppose que le taux swap forward suit la dynamique de Black présentée ci-dessus.

Notons $S_{\alpha, \beta}(t)$ le taux swap forward couvrant la période entre $T_{\alpha}$ et $T_{\beta}$ :

$$
S_{\alpha, \beta}(t)=\frac{P\left(t, T_{\alpha}\right)-P\left(t, T_{\beta}\right)}{\sum_{j=\alpha+1}^{\beta} \tau_{j} P\left(t, T_{j}\right)}
$$

Le prix d'une swaption européenne payeuse de ténor $T_{\alpha}-T_{\beta}$, de nominal $N$ et de prix d'exercice $K$ est donné par (Brigo et Mercurio [2007]):

$$
P S^{\text {Black }}\left(0, T, \tau, N, K, \sigma_{\alpha, \beta}\right)=N . B l\left(K, S_{\alpha, \beta}(0), \sigma_{\alpha, \beta} \sqrt{T_{\alpha}}, 1\right) \sum_{i=\alpha+1}^{\beta} P\left(0, T_{i}\right) \tau_{i}
$$

Avec:

- $T=\left\{T_{0}, \ldots, T_{M}\right\}$ un ensemble discret de dates;

- $\tau=\left\{\tau_{1}, \ldots, \tau_{n}\right\}$ un ensemble tel que $\tau_{i}$ représente la différence entre $T_{i-1}$ et $T_{i}$;

- $P\left(0, T_{i}\right)$ est le prix d'une obligation zéro-coupon observé en $t=0$ pour l'échéance $T_{i}$;

- $B l(K, F, v, \omega)=F \omega \Phi\left(\omega d_{1}(K, F, v)\right)-K \omega \Phi\left(\omega d_{2}(K, F, v)\right)$; 
- $\Phi$ la fonction de distribution gaussienne standard;

- $d_{1}(K, F, v)=\frac{\ln \left(\frac{F}{K}\right)+\frac{v^{2}}{2}}{v}$

- $d_{2}(K, F, v)=\frac{\ln \left(\frac{F}{K}\right)-\frac{v^{2}}{2}}{v}$;

- $\sigma_{\alpha, \beta}$ est la volatilité (généralement extraite des cours du marché).

Notons que le paramètre de volatilité implicite $\sigma_{\alpha, \beta}$ est totalement déterminé à partir du prix d'exercice, des prix de marché des obligations zéro-coupon sans risque et des prix des swaptions. En d'autres termes, étant donnés un prix d'exercice et une courbe de taux, la correspondance entre les prix des swaptions et les volatilités implicites est une bijection.

\subsubsection{Le modèle de Black et les taux négatifs}

Le modèle de Black décrit dans les sections précédentes est un modèle log-normal. L'utilisation de ce modèle dans l'état ne peut s'opérer dans un environnement économique caractérisé par des taux négatifs.

Afin de rendre le modèle de Black cohérent avec des taux négatifs, un facteur de décalage des taux est introduit. Ce facteur est appelé "shift" ou "displacement factor". On parle alors du modèle de Black décalé ou du displaced Black model. La dynamique du modèle s'écrit en effet:

$$
d S_{\alpha, \beta}=\left(S_{\alpha, \beta}+a\right) \cdot \sigma_{\alpha, \beta}^{(a)} \cdot d W_{t}^{\alpha, \beta}
$$

Le facteur de translation étant a. Ce facteur est choisi de telle sorte que le prix d'exercice augmenté de $a$ et les valeurs observées de $S_{\alpha, \beta}+a$ soient toujours positifs.

Le modèle de Black décalé ne prend en compte que les taux négatifs supérieurs à $-a$. En pratique, ce facteur est choisi $a$ priori. Une attention particulière doit être accordée au processus du choix du facteur de décalage. En effet la valeur du facteur de décalage:

- devrait être suffisante pour éviter que les grandeurs $S_{\alpha, \beta}+a$ et $K+a$ ne passent en dessous de zéro;

- mais ne devrait pas être extrêmement élevé car dans ce cas le taux forward peut avoir des valeurs négatives significatives. 
Par ailleurs, en définissant $\bar{S}_{\alpha, \beta}=S_{\alpha, \beta}+a$ et $\bar{K}=K+a$ la formulation du modèle Black décalé est équivalente à celle du modèle de Black. Par conséquent, les formules de valorisation des caps, floors et swaptions présentées dans les sections précédentes sont valables pour le modèle de Black décalé en remplaçant $S_{\alpha, \beta}$ par $\bar{S}_{\alpha, \beta}, K$ par $\bar{K}$ et $\sigma_{\alpha, \beta}$ $\operatorname{par} \sigma_{\alpha, \beta}^{(a)}$.

Cependant, il est important de noter que les volatilités implicites aux prix dépendent du facteur de décalage. En effet, pour un prix d'exercice donné, quatre grandeurs entrent dans la construction du modèle de Black décalé:

1. La courbe des taux;

2. Le prix du dérivé (caps, floors ou swaption);

3. Le facteur de décalage;

4. Les volatilités implicites.

Donc, pour une courbe de taux donnée, il faut disposer des valeurs de deux variables parmi les variables 2,3 et 4, pour déduire la troisième variable. Quand les prix sont définis et extraits des marchés financiers, les volatilités implicites dépendent systématiquement du facteur de décalage.

Ainsi, l'utilisation des volatilités implicites aux prix du marché dans un processus de valorisation de produits dérivés par le modèle de Black décalé dans un environnement à taux négatifs ne peut se faire sans précision de la valeur du facteur de décalage utilisé pour générer ces volatilités. L'utilisation de volatilités décalées cohérentes avec le facteur de décalage et la courbe des taux permet de reproduire les prix de marché.

\subsubsection{Modèle Hull \& White}

\subsubsection{Dynamique du modèle}

Le modèle Hull \& White généralise le modèle de Vasicek [1977] et introduit une moyenne à long terme fonction du temps permettant au modèle de reproduire la courbe des taux d'intérêt par terme anticipée par le marché. 
Hull \& White [1990] supposent en effet que le taux d'intérêt instantané sous la probabilité risque neutre suit la dynamique suivante:

$$
d r(t)=[\vartheta(t)-a(t) r(t)] d t+\sigma(t) d W(t)
$$

où $\vartheta, a$ and $\sigma$ sont des fonctions déterministes.

Afin d'éviter la complexité qu'engendre le calibrage d'une surface de volatilité implicite au modèle ci-dessus, nous avons fait le choix de retenir le modèle suivant présenté par Hull et White [1994]:

$$
d r(t)=[\vartheta(t)-a \cdot r(t)] d t+\sigma \cdot d W(t)
$$

La fonction $\vartheta$ est choisie pour que le modèle reproduise la structure par terme des taux d'intérêt actuellement anticipés par le marché. Lea calibrage du modèle Hull \& White consiste donc à estimer les paramètres $a$ et $\sigma$.

Il est noté que le modèle Hull \& White est un cas particulier de la famille des modèles HJM dans lequel on suppose que le processus de volatilité vérifie la relation suivante:

$$
\sigma(t, T)=\sigma \exp (-a(T-t))
$$

Cette forme est facilement interprétable: plus on se rapproche de l'échéance, plus la volatilité diminue.

\subsubsection{Solution de l'équation différentielle}

Soit $f^{M}(0, T)$ le taux forward instantané du marché observé à la date 0 pour la maturité $T$ :

$$
f^{M}(0, T)=-\frac{\partial \ln \left(P^{M}(0, T)\right)}{\partial T}
$$

$P^{M}(0, T)$ étant le prix d'une obligation zéro-coupon observée à $\mathrm{t}=0$ dont la maturité est $T$.

La moyenne à long terme s'écrit alors :

$$
\vartheta(t)=\frac{\partial f^{M}(0, t)}{\partial T}+a f^{M}(0, t)+\frac{\sigma^{2}}{2 a}\left(1-e^{-2 a t}\right)
$$


où $\frac{\partial f M}{\partial T}$ est la dérivée partielle du taux forward $f^{M}$ suivant la deuxième variable.

La solution de l'équation différentielle présentée par Hull \& White s'écrit:

$$
r(t)=r(s) e^{-a(t-s)}+\alpha(t)-\alpha(s) e^{-a(t-s)}+\sigma \int_{s}^{t} e^{-a(t-u)} d W(u)
$$

où $\alpha(t)=f^{M}(0, t)+\frac{\sigma^{2}}{2 a^{2}}\left(1-e^{-a t}\right)^{2}$.

Ainsi, la variable $r(t)$ est distribuée normalement conditionnellement à $F_{s}$. La moyenne et la variance s'écrivent:

$$
\begin{gathered}
E\left\{r(t) \mid F_{s}\right\}=r(s) e^{-a(t-s)}+\alpha(t)-\alpha(s) e^{-a(t-s)} \\
\operatorname{Var}\left\{r(t) \mid F_{s}\right\}=\frac{\sigma^{2}}{2 a}\left(1-e^{-2 a(t-s)}\right)
\end{gathered}
$$

\subsubsection{Prix d'une obligation zéro-coupon}

Le prix d'une obligation zéro-coupon observée à l'instant $t$ et payant une unité monétaire à l'échéance $T$ s'écrit:

$$
P(t, T)=E\left(e^{-\int_{t}^{T} r(s) d s} \mid r(t)\right)
$$

Notons que la variable $\int_{t}^{T} r(s) d s \mid r(t)$ est distribuée suivant une loi normale:

$\int_{t}^{T} r(s) d s \mid r(t) \sim N\left(B(t, T)[r(t)-\alpha(t)]+\ln \frac{P^{M}(0, t)}{P^{M}(0, T)}+\frac{1}{2}[V(0, T)-V(0, t)], V(t, T)\right)$

Avec:

- $B(t, T)=\frac{1}{a}\left(1-e^{-a(T-t)}\right)$;

- $V(t, T)=\frac{\sigma^{2}}{a^{2}}\left(T-t+\frac{2}{a} e^{-a(T-t)}-\frac{1}{2 a} e^{-2 a(T-t)}-\frac{3}{2 a}\right)$. 
Soit: $A(t, T)=\frac{P^{M}(0, T)}{P^{M}(0, t)} \exp \left(B(t, T) f^{M}(0, t)-\frac{\sigma^{2}}{4 a}\left(1-e^{-2 a t}\right) B(t, T)^{2}\right)$.

Alors le prix d'une obligation zéro coupon s'écrit: $P(t, T)=$ $A(t, T) e^{-B(t, T) r(t)}$.

Notons que la distribution du prix d'une obligation zéro-coupon est log-normale.

\subsubsection{Calibrage du modèle Hull \& White sur les caps}

Le modèle de Hull \& White offre la possibilité de valoriser les caps et les floors par des formules fermées. Notons $T=\left\{t_{0}, t_{1}, \ldots, t_{n}\right\}$ l'ensemble des maturités de paiements des caps ou des floors. Soit $\tau_{i}$ la différence entre $t_{i-1}$ et $t_{i}$.

Le prix à l'instant $t<t_{0}$ du cap de prix d'exercice $X$, de valeur nominale $N$ et défini sur l'ensemble $T=\left\{t_{0}, t_{1}, \ldots, t_{n}\right\}$ est donné par:

$\operatorname{Cap}(t, T, N, X)=N \sum_{i=1}^{n}\left(P\left(t, t_{i-1}\right) \Phi\left(-h_{i}+\sigma_{p}^{i}\right)-\left(1+X \tau_{i}\right) P\left(t, t_{i}\right) \Phi\left(-h_{i}\right)\right)$

Le prix du floor est donné par:

$\operatorname{Flr}(t, T, N, X)=N \sum_{i=1}^{n}\left(\left(1+X \tau_{i}\right) P\left(t, t_{i}\right) \Phi\left(h_{i}\right)-P\left(t, t_{i-1}\right) \Phi\left(h_{i}-\sigma_{p}^{i}\right)\right)$

Avec:

- $\sigma_{p}^{i}=\sigma \sqrt{\frac{1-e^{-2 a\left(t_{i-1}-t\right)}}{2 a}} B\left(t_{i-1}, t_{i}\right)$;

- $h_{i}=\frac{1}{\sigma_{p}^{i}} \ln \frac{P\left(t, t_{i}\right)\left(1+X \tau_{i}\right)}{P\left(t, t_{i-1}\right)}+\frac{\sigma_{p}^{i}}{2}$;

- $\Phi$ est la fonction de répartition gaussienne.

Notons :

- $\theta=(a, \sigma)$ : les paramètres du modèle;

- Prix ${ }_{i}^{\text {black }}$ : le prix d'un cap $i$ valorisé par le marché via la formule de Black;

- $\operatorname{Cap}_{i}^{H W}(\theta)$ : le prix d'un cap $i$ valorisé par le modèle Hull \& White. 
Les paramètres du modèle sont déduits par l'optimisation suivante sur l'ensemble des caps retenus pour le calibrage ( $d$ est une distance):

$$
\hat{\theta}=\underset{\theta}{\operatorname{argmin}}\left(d\left(\left(\text { Prix }_{i}^{\text {black }}\right)_{i},\left(\operatorname{Cap}_{i}^{H W}(\theta)\right)_{i}\right)\right)
$$

Les prix valorisés par le modèle de Black à partir des volatilités implicites jouent ici le rôle de prix de marché reconstitués. Cette reconstitution par le modèle de Black a, entre autres, deux avantages. Elle permet en effet de:

- Retrouver les prix observés du marché ayant servi pour le calcul des volatilités implicites;

- Produire des prix cohérents avec les prix de marché quand les volatilités implicites utilisées sont extraites de modèles de volatilités calibrés sur les volatilités du marché (exemple: reconstruction de la nappe de volatilités sur des dates supplémentaires à partir des volatilités de marché).

Si l'on dispose des prix directement observables, le modèle de Hull \& White peut être calibré directement sur ces prix de marché en réécrivant la fonction d'optimisation comme suit:

$$
\hat{\theta}=\underset{\theta}{\operatorname{argmin}}\left(d\left(\left(\text { Prix }_{i}^{\text {market }}\right)_{i},\left(\operatorname{Cap}_{i}^{H W}(\theta)\right)_{i}\right)\right)
$$

\subsubsection{Calibrage du modèle Hull \& White sur des swaptions européennes}

Pour calibrer le modèle Hull \& White sur des swaptions, nous avons choisi une méthode basée sur la génération d'arbres trinomiaux. Brigo et Mercurio [2007] présentent le cadre théorique et pratique de génération d'arbre avec le modèle Hull et White.

Le payoff actualisé d'une swaption européenne payeuse de nominal $N$ de date d'exercice $T_{\alpha}$, de maturité $T_{\beta}$ et évaluée à la date $t=0$ est égal à:

$$
f_{\alpha, \beta}(a, \sigma)=N . D\left(t, T_{\alpha}\right) \sum_{i=\alpha+1}^{\beta} P\left(T_{\alpha}, T_{i}\right) \tau_{i}\left(F\left(T_{\alpha}, T_{i-1}, T_{i}\right)-K\right)^{+}
$$


Avec:

- $K$ est le prix d'exercice;

- $D\left(t, T_{\alpha}\right)$ est le déflateur calculé à $t$ pour l'échéance $T_{\alpha}$;

- $P\left(T_{\alpha}, T_{i}\right)$ est le prix du zéro-coupon calculé à $T_{\alpha}$ pour l'échéance $T_{i}$;

- $F\left(T_{\alpha}, T_{i-1}, T_{i}\right)$ est le taux forward calculé à $T_{\alpha}$ pour la période entre $T_{i-1}$ et $T_{i}$;

- $\tau_{i}$ est la différence entre $T_{i-1}$ et $T_{i}$;

- $a$ et $\sigma$ sont les paramètres du modèle Hull \& White.

Notons :

- $\theta=(a, \sigma)$ : les paramètres du modèle;

- Prix ${ }_{i}^{\text {black }}$ : le prix d'une swaption $i$ valorisée par le marché via la formule de Black;

- $f_{i}^{H W}(\theta)$ : le prix d'une swaption $i$ valorisée par le modèle Hull \& White.

Les paramètres du modèle sont déduits par l'optimisation suivante sur l'ensemble des swaptions retenues pour le calibrage ( $d$ est une distance):

$$
\hat{\theta}=\underset{\theta}{\operatorname{argmin}}\left(d\left(\left(\text { Prix }_{i}^{\text {black }}\right)_{i},\left(f_{i}^{H W}(\theta)\right)_{i}\right)\right)
$$

Cette optimisation consiste en un algorithme récursif basé sur l'approche de Newton. À chaque itération, un arbre est généré et un prix de swaption est évalué à chaque nœud puis actualisé par le déflateur correspondant. La distance entre les prix de marché et les prix théoriques est ensuite calculée. Les itérations peuvent s'arrêter sur un optimum local, d'où la nécessité d'initialiser l'algorithme d'optimisation par plusieurs points.

Les prix valorisés par le modèle de Black à partir des volatilités implicites jouent ici le rôle de prix de marché reconstitués. Cette reconstitution permet de retrouver des prix cohérents avec les prix observés. 
Si l'on dispose des prix directement observables, le modèle de Hull \& White peut être calibré directement sur ces prix de marché en réécrivant la fonction d'optimisation comme suit:

$$
\hat{\theta}=\underset{\theta}{\operatorname{argmin}}\left(d\left(\left(\text { Prix }_{i}^{\text {market }}\right)_{i},\left(f_{i}^{H W}(\theta)\right)_{i}\right)\right)
$$

\subsubsection{Le modèle Hull \& White et les taux négatifs}

Le modèle Hull \& White est un modèle normal. Il permet donc de générer des taux négatifs.

Si l'on dispose des prix directement observables, le modèle de Hull \& White peut être calibré directement sur ces prix de marché.

L'utilisation des volatilités de Black implicites du marché pour le calibrage du modèle Hull \& White dans un environnement économique à taux négatifs, nécessite l'utilisation du modèle de Black décalé.

Le calibrage du modèle de Hull \& White sur des caps, des floors ou des swaptions valorisés par le modèle de Black à partir des volatilités implicites peut se présenter comme suit:

- Étape 1: calibrage du modèle de Black décalé consistant à définir, pour une courbe de taux sans risque donnée, un facteur de décalage et à extraire les volatilités implicites cohérentes avec la valeur du facteur de décalage choisi en amont comme précisé dans la section 2.1.1.3;

- Étape 2: valorisation du produit dérivé dans le cadre du modèle de Black décalé par la formule de Black comme présenté dans la section 2.1.1.3. Cette étape permet de disposer des prix de marché des actifs dérivés cohérents, a priori, avec les valeurs observées car les volatilités implicites et le facteur de décalage sont par construction cohérents avec ces observations;

- Étape 3: calibrer le modèle Hull \& White en utilisant les mêmes démarches présentées dans les sections 2.1.2.4 et 2.1.2.5.

Les paramètres du modèle Hull \& White calibrés ainsi sont cohérents avec les valeurs observées et la courbe de taux sans risques dans un environnement à taux négatifs. 
2.1.3 Modèle gaussien linéaire à deux facteurs $(\mathrm{G} 2++)$

\subsubsection{Dynamique du modèle}

Dans cette section, nous présentons un modèle de taux d'intérêt où le processus instantané de taux court est donné par la somme de deux facteurs gaussiens corrélés plus une fonction déterministe. Cette fonction permet la reproduction de la structure par terme des taux d'intérêt sans risque. Le modèle permet de valoriser, par des formules fermées, les obligations zéro-coupons, les caps, les floors et les swaptions. Il est noté par ailleurs que ce modèle permet de générer des taux négatifs.

Le taux d'intérêt instantané sous la probabilité risque neutre suit la dynamique suivante:

$$
r(t)=x(t)+y(t)+\phi(t)
$$

- Notons : $r(0)=r_{0}$;

- Les deux processus: $\{x(t): t \geq 0\}$ et $\{y(t): t \geq 0\}$ sont définis comme suit:

$$
\begin{aligned}
& d x(t)=-a x(t) d t+\sigma d W_{1}(t) \text { et } x(0)=0 \\
& d y(t)=-b y(t) d t+\eta d W_{2}(t) \text { et } y(0)=0
\end{aligned}
$$

- $W_{1}$ et $W_{2}$ sont deux mouvements browniens corrélés: $d W_{1}(t) d W_{2}(t)=\rho d t$ et $-1 \leq \rho \leq 1 ;$

- Les paramètres $r_{0}, a, b, \sigma, \eta$ sont des constantes positives;

- La fonction $\phi$ est déterministe, définie sur toute la période de projection et $\phi(0)=r_{0}$.

\subsubsection{Solution de l'équation différentielle}

Soit $f^{M}(0, T)$ le taux forward instantané du marché observé à la date 0 pour la maturité $T$ :

$$
f^{M}(0, T)=-\frac{\partial \ln \left(P^{M}(0, T)\right)}{\partial T}
$$


Et soit $F_{t}$ le sigma-ensemble généré par la paire $(x, y)$ jusqu'à la date $t$.

La solution de l'équation différentielle du modèle G2++ s'écrit:

$$
r(t)=x(s) e^{-a(t-s)}+y(s) e^{-b(t-s)}+\sigma \int_{s}^{t} e^{-a(t-u)} d W_{1}(u)+\eta \int_{s}^{t} e^{-b(t-u)} d W_{2}(u)+\phi(t)
$$

Donc le taux instantané $r(t)$ suit une loi normale conditionnellement à $F_{s}$. Sa moyenne et sa variance sont respectivement:

- $E\{r(t) \mid F s\}=x(s) e^{-a(t-s)}+y(s) e^{-b(t-s)}+\phi(t)$;

- $\operatorname{Var}\{r(t) \mid F s\}=\frac{\sigma^{2}}{2 a}\left(1-e^{-2 a(t-s)}\right)+\frac{\eta^{2}}{2 b}\left(1-e^{-2 b(t-s)}\right)+2 \rho \frac{\sigma \eta}{a+b}\left(1-e^{-(a+b)(t-s)}\right)$.

En particulier: $r(t)=\sigma \int_{0}^{t} e^{-a(t-u)} d W_{1}(u)+\eta \int_{0}^{t} e^{-b(t-u)} d W_{2}(u)+\phi(t)$.

Par ailleurs, la fonction $\phi$ permet de reproduire la structure par terme observée si et seulement si, pour chaque $T$ :

$\phi(T)=f^{M}(0, T)+\frac{\sigma^{2}}{2 a^{2}}\left(1-e^{-a T}\right)^{2}+\frac{\eta^{2}}{2 b^{2}}\left(1-e^{-b T}\right)^{2}+\rho \frac{\sigma \eta}{a b}\left(1-e^{-a T}\right)\left(1-e^{-b T}\right)$

\subsubsection{Prix d'une obligation zéro-coupon}

Le prix d'une obligation zéro-coupon observé à l'instant $t$ et payant une unité monétaire à l'échéance $T$ s'écrit:

$$
P(t, T)=E\left(e^{-\int_{t}^{T} r(s) d s} \mid F_{t}\right)
$$

Le prix d'une obligation zéro-coupon valorisée par le modèle G2++ s'écrit (Brigo et Mercurio [2007]):

$$
P(t, T)=A(t, T) \exp (-B(a, t, T) x(t)-B(b, t, T) y(t))
$$


Avec:

- $A(t, T)=\frac{P^{M}(0, T)}{P^{M}(0, t)} \exp \left(\frac{1}{2}(V(t, T)-V(0, T)+V(0, t))\right)$;

- $B(z, t, T)=\frac{1-e^{-z(T-t)}}{z}$;

- $V(t, T)=\frac{\sigma^{2}}{a^{2}}\left(T-t+\frac{2}{a} e^{-a(T-t)}-\frac{1}{2 a} e^{-2 a(T-t)}-\frac{3}{2 a}\right)+$

$$
\begin{aligned}
& \frac{\eta^{2}}{b^{2}}\left(T-t+\frac{2}{b} e^{-b(T-t)}-\frac{1}{2 b} e^{-2 b(T-t)}-\frac{3}{2 b}\right)+ \\
& 2 \rho \frac{\sigma \eta}{a b}\left(T-t+\frac{e^{-a(T-t)}-1}{a}+\frac{e^{-b(T-t)}-1}{b}-\frac{e^{-(a+b)(T-t)}-1}{a+b}\right) ;
\end{aligned}
$$

- $P^{M}(0, T)$ le prix d'une obligation zéro-coupon observée à $t=0$ pour la maturité $T$.

Notons que la distribution du prix d'une obligation zéro-coupon est log-normale.

\subsubsection{Calibrage du modèle G2++ sur les caps}

Notons $T=\left\{T_{0}, T_{1}, \ldots, T_{n}\right\}$ l'ensemble des dates de paiement des caps/floors et $T_{0}$ le temps d'initialisation. Soit $\tau=\left\{\tau_{1}, \ldots, \tau_{n}\right\}$ tel que $\tau_{i}$ est la différence entre $T_{i-1}$ et $T_{i}$. Le prix à l'instant $t<T_{0}$ du cap de prix d'exercice $X$ et de valeur nominale $N$ est donné par:

$\operatorname{Cap}(t, T, \tau, N, X)=$

$$
\begin{aligned}
& \sum_{i=1}^{n}\left(-N\left(1+X \tau_{i}\right) P\left(t, T_{i}\right) \Phi\left(\frac{\ln \frac{P\left(t, T_{i-1}\right)}{\left(1+X \tau_{i}\right) P\left(t, T_{i}\right)}}{\Sigma\left(t, T_{i-1}, T_{i}\right)}-\frac{1}{2} \Sigma\left(t, T_{i-1}, T_{i}\right)\right)\right. \\
& \quad+P\left(t, T_{i-1}\right) N \Phi\left(\frac{\ln \frac{P\left(t, T_{i-1}\right)}{\left(1+X \tau_{i}\right) P\left(t, T_{i}\right)}}{\Sigma\left(t, T_{i-1}, T_{i}\right)}+\frac{1}{2} \Sigma\left(t, T_{i-1}, T_{i}\right)\right)
\end{aligned}
$$


Le prix du floor s'écrit:

$$
\begin{aligned}
& F \operatorname{lr}(t, T, \tau, N, X)= \\
& \begin{array}{l}
\sum_{i=1}^{n}\left(N\left(1+X \tau_{i}\right) P\left(t, T_{i}\right) \Phi\left(\frac{\ln \frac{\left(1+X \tau_{i}\right) P\left(t, T_{i}\right)}{P\left(t, T_{i-1}\right)}}{\Sigma\left(t, T_{i-1}, T_{i}\right)}+\frac{1}{2} \Sigma\left(t, T_{i-1}, T_{i}\right)\right)\right. \\
\left.\quad-P\left(t, T_{i-1}\right) N \Phi\left(\frac{\ln \frac{\left(1+X \tau_{i}\right) P\left(t, T_{i}\right)}{P\left(t, T_{i-1}\right)}}{\Sigma\left(t, T_{i-1}, T_{i}\right)}-\frac{1}{2} \Sigma\left(t, T_{i-1}, T_{i}\right)\right)\right)
\end{array}
\end{aligned}
$$

Le paramètre $\sum$ est positif et défini comme suit:

$$
\begin{aligned}
\sum(t, T, S)^{2}= & \frac{\sigma^{2}}{2 a^{3}}\left(1-e^{-a(S-T)}\right)^{2}\left(1-e^{-2 a(T-t)}\right)+\frac{\eta^{2}}{2 b^{3}}\left(1-e^{-b(S-T)}\right)^{2}\left(1-e^{-2 b(T-t)}\right) \\
& +2 \rho \frac{\sigma \eta}{a b(a+b)}\left(1-e^{-a(S-T)}\right)\left(1-e^{-b(S-T)}\right)\left(1-e^{-(a+b)(T-t)}\right)
\end{aligned}
$$

Notons :

- $\theta=(a, \sigma, b, \eta, \rho)$ : les paramètres du modèle;

- Prix ${ }_{i}^{\text {black }}$ : le prix d'un cap $i$ valorisé par le marché via la formule de Black;

- $C a p_{i}^{G 2++}(\theta)$ : le prix d'un cap $i$ valorisé par le modèle G2++.

Les paramètres du modèle sont déduits par l'optimisation suivante sur l'ensemble des caps retenus pour le calibrage ( $d$ est une distance):

$$
\hat{\theta}=\underset{\theta}{\operatorname{argmin}}\left(d\left(\left(\text { Prix }_{i}^{\text {black }}\right)_{i},\left(\operatorname{Cap}_{i}^{G 2++}(\theta)\right)_{i}\right)\right)
$$

Les prix valorisés par le modèle de Black à partir des volatilités implicites jouent ici le rôle de prix de marché reconstitués. Cette reconstitution permet de retrouver des prix cohérents avec les prix observés. 
Si l'on dispose des prix directement observables, le modèle G2++ peut être calibré directement sur ces prix de marché en réécrivant la fonction d'optimisation comme suit:

$$
\hat{\theta}=\underset{\theta}{\operatorname{argmin}}\left(d\left(\left(\text { Prix }_{i}^{\text {market }}\right)_{i},\left(\operatorname{Cap}_{i}^{G 2++}(\theta)\right)_{i}\right)\right)
$$

\subsubsection{Calibrage du modèle G2++ sur les swaptions européennes}

Considérons une swaption Européenne de prix d'exercice $X$, de date d'exercice $T$ et de nominal $N$, donnant au détenteur de l'option le droit d'entrer à l'instant $t_{0}=T$ dans un swap de taux dont les dates de paiement sont $\mathfrak{I}=\left\{t_{1}, \ldots, t_{n}\right\}, t_{1}>T$, où il paie un taux fixe $X$ contre un taux variable. Notons: $\tau_{i}$ la différence entre $t_{i-1}$ et $t_{i}, c_{i}=\tau_{i} X$ et $c_{n}=1+\tau_{n} X$.

Le prix théorique sans opportunité d'arbitrage d'une swaption est donné par l'intégrale suivante:

$$
\begin{aligned}
E S(t= & 0, T, \mathfrak{I}, N, X, \omega) \\
& =N \omega P(0, T) \int_{-\infty}^{+\infty} \frac{e^{-\frac{1}{2}\left(\frac{x-\mu_{x}}{\sigma_{x}}\right)^{2}}}{\sigma_{x} \sqrt{2 \pi}}\left[\Phi\left(-\omega h_{1}(x)\right)-\sum_{i=1}^{n} \lambda_{i}(x) e^{\kappa_{i}(x)} \Phi\left(-\omega h_{2}(x)\right)\right] d x
\end{aligned}
$$

Avec:

- $\omega=1(\omega=-1)$ pour une swaption payeuse (receveuse);

- $h_{1}(x)=\frac{\bar{y}-\mu_{y}}{\sigma_{y} \sqrt{1-\rho_{x y}^{2}}}-\frac{\rho_{x y}\left(x-\mu_{x}\right)}{\sigma_{x} \sqrt{1-\rho_{x y}^{2}}}$;

- $h_{2}(x)=h_{1}(x)+B\left(b, T, t_{i}\right) \sigma_{y} \sqrt{1-\rho_{x y}^{2}}$;

- $\lambda_{i}(x)=c_{i} A\left(T, t_{i}\right) e^{-B\left(a, T, t_{i}\right) x}$;

- $\kappa_{i}(x)=-B\left(b, T, t_{i}\right)\left(\mu_{y}-\frac{1}{2}\left(1-\rho_{x y}^{2}\right) \sigma_{y}^{2} B\left(b, T, t_{i}\right)+\frac{\rho_{x y} \sigma_{y}\left(x-\mu_{x}\right)}{\sigma x}\right.$;

- $\bar{y}=\bar{y}(x)$ est l'unique solution de l'équation suivante:

$$
\sum_{i=1}^{n} c_{i} A\left(T, t_{i}\right) e^{-B\left(a, T, t_{i}\right) x-B\left(b, T, t_{i}\right) \bar{y}}=1
$$

- $\mu_{x}=-M_{x}^{T}(0, T)$;

- $\mu_{y}=-M_{y}^{T}(0, T)$; 
- $\sigma_{x}=\sigma \sqrt{\frac{1-e^{-2 a T}}{2 a}}$

- $\sigma_{y}=\sigma \sqrt{\frac{1-e^{-2 b T}}{2 b}}$;

- $\rho_{x y}=\frac{\rho \sigma \eta}{(a+b) \sigma_{x} \sigma_{y}}\left(1-e^{-(a+b) T}\right)$;

- $M_{x}^{T}(s, t)=\left(\frac{\sigma^{2}}{a^{2}}+\rho \frac{\sigma \eta}{a b}\right)\left(1-e^{-a(t-s)}\right)-\frac{\sigma^{2}}{2 a^{2}}\left(e^{-a(T-t)}-e^{-a(T+t-2 s)}\right)$

$$
-\frac{\rho \sigma \eta}{b(a+b)}\left(e^{-b(T-t)}-e^{-b T-a t+(a+b) s}\right) \text {; }
$$

- $M_{y}^{T}(s, t)=\left(\frac{\eta^{2}}{b^{2}}+\rho \frac{\sigma \eta}{a b}\right)\left(1-e^{-b(t-s)}\right)-\frac{\eta^{2}}{2 b^{2}}\left(e^{-b(T-t)}-e^{-b(T+t-2 s)}\right)$

$$
-\frac{\rho \sigma \eta}{a(a+b)}\left(e^{-a(T-t)}-e^{-a T-b t+(a+b) s}\right) .
$$

La formule de valorisation de swaption n'est pas totalement fermée dans la mesure où l'intégrale ci-dessus n'a pas de bornes finies. On peut noter par ailleurs que l'intégrale est calculée sur une variable distribuée suivant la loi normale: $X \sim N\left(\mu_{x}, \sigma_{x}^{2}\right)$. Ainsi, pour les applications numériques de ce travail, l'intégrale est calculée par la méthode des trapèzes sur l'intervalle $\left[\mu_{x}-10 \sigma_{x}, \mu_{x}+10 \sigma_{x}\right]$.

Notons :

- $\theta=(a, \sigma, b, \eta, \rho)$ : les paramètres du modèle;

- Prix ${ }_{i}^{\text {black }}$ : le prix d'une swaption $i$ valorisée par le marché via la formule de Black;

- $E S_{i}^{G 2++}(\theta)$ : le prix d'une swaption $i$ valorisée par le modèle G2++.

Les paramètres du modèle sont déduits par l'optimisation suivante sur l'ensemble des swaptions retenues ( $d$ est une distance):

$$
\hat{\theta}=\underset{\theta}{\operatorname{argmin}}\left(d\left(\left(\text { Prix }_{i}^{\text {black }}\right)_{i},\left(E S_{i}^{G 2++}(\theta)\right)_{i}\right)\right)
$$

Les prix valorisés par le modèle de Black à partir des volatilités implicites jouent ici le rôle de prix de marché reconstitués. Cette reconstitution permet de retrouver des prix cohérents avec les prix observés. 
Si l'on dispose des prix directement observables, le modèle G2++ peut être calibré directement sur ces prix de marché en réécrivant la fonction d'optimisation comme suit:

$$
\hat{\theta}=\underset{\theta}{\operatorname{argmin}}\left(d\left(\left(\text { Prix }_{i}^{\text {market }}\right)_{i},\left(E S_{i}^{G 2++}(\theta)\right)_{i}\right)\right)
$$

\subsubsection{Le modèle G2++ et les taux négatifs}

Le modèle G2++ est un modèle normal. Il permet donc de générer des taux négatifs.

Si l'on dispose des prix directement observables, le modèle G2++ peut être calibré directement sur ces prix de marché.

L'utilisation des volatilités de Black implicites du marché pour le calibrage du modèle G2++ dans un environnement économique à taux négatifs, nécessite l'utilisation du modèle de Black décalé et suit le même processus présenté dans la section 2.1.2.6.

\subsubsection{Libor Market Model (LMM)}

\subsubsection{Dynamique du modèle}

Un modèle de taux de marché décrit directement la dynamique des taux négociables contrairement aux modèles Hull \& White et G2++. Ces derniers modélisent en effet un processus caché sous-jacent aux prix des titres d'intérêt: le taux court instantané.

Considérons un ensemble $E=\left\{T_{0}, \ldots, T_{M}\right\}$ à partir duquel sont construites des paires de dates d'échéance $\left(T_{i-1}, T_{i}\right)$. Soit $\left\{\tau_{0}, \ldots, \tau_{M}\right\}$ un ensemble tel que $\tau_{i}$ est la différence entre $T_{i-1}$ et $T_{i}$. On note $T_{-1}=0$. Le taux forward noté $F_{k}(t)=F\left(t, T_{k-1}, T_{k}\right), k \in\{1, \ldots, M\}$ est égale au taux spot pour $t=T_{k-1}: F_{k}\left(T_{k-1}\right)=L\left(T_{k-1}, T_{k}\right)$ où $L(S, T)$ désigne le taux spot observé à la date $S$ pour l'échéance $T$.

Considérons maintenant la mesure de probabilité $Q^{k}$ associée au numéraire $P\left(\bullet, T_{k}\right)$, soit au prix de l'obligation zéro-coupons dont la maturité coïncide avec la maturité du taux à terme. $Q^{k}$ est souvent appelé la mesure forward (ajustée) pour la maturité $T_{k}$.

Par définition: $F_{k}(t) P\left(t, T_{k}\right)=\frac{\left[P\left(t, T_{k-1}\right)-P\left(t, T_{k}\right)\right]}{\tau_{k}}$. 
Et donc: $P\left(t, T_{k}\right)=\frac{P\left(t, T_{k-1}\right)}{1+\tau_{k} F_{k}(t)}$.

La dynamique de $F_{k}$ sous la probabilité $Q^{k}$ est donnée par:

$$
d F_{k}(t)=\sigma_{k}(t) F_{k}(t) d Z_{k}(t) ; t \leq T_{k-1}
$$

La variable $Z_{i}(t)$ est un mouvement Brownian sous $Q^{i}$ tel que: $d Z_{i}(t) d Z_{j}(t)=\rho_{i, j} d t$.

Plus généralement, pour tout entier naturel strictement positif $i$, la dynamique du taux forward sous $Q^{i}$ s'écrit (cf. Brigo et Mercurio [2007]):

- Pour: $i<k$ et $t \leq T_{i}$ :

$$
d F_{k}(t)=\sigma_{k}(t) F_{k}(t) \sum_{j=i+1}^{k} \rho_{k, j} \frac{\tau_{j} \sigma_{j}(t) F_{j}(t)}{1+\tau_{j} F_{j}(t)} d t+\sigma_{k}(t) F_{k}(t) d Z_{k}(t)
$$

- Pour: $i=k$ et $t \leq T_{k-1}$ :

$$
d F_{k}(t)=\sigma_{k}(t) F_{k}(t) d Z_{k}(t) ; t \leq T_{k-1}
$$

- Pour: $i>k$ et $t \leq T_{k-1}$ :

$$
d F_{k}(t)=-\sigma_{k}(t) F_{k}(t) \sum_{j=k+1}^{i} \rho_{k, j} \frac{\tau_{j} \sigma_{j}(t) F_{j}(t)}{1+\tau_{j} F_{j}(t)} d t+\sigma_{k}(t) F_{k}(t) d Z_{k}(t)
$$

\subsubsection{Solution de l'équation différentielle}

Notons :

- $\beta$ une fonction de $t$ tel que: $\beta(t)=m$ si $T_{m-2}<t \leq T_{m-1}$ et $m \geq 1$. Donc $\left.t \in] T_{\beta(t)-2}, T_{\beta(t)-1}\right]$;

- $B(T)=\exp \left(\int_{0}^{T} r_{t} d t\right)$

Soit :

$$
B_{d}(t)=\frac{P\left(t, T_{\beta(t)-1}\right)}{\prod_{j=0}^{\beta(t)-1} P\left(T_{j-1}, T_{j}\right)}=P\left(t, T_{\beta(t)-1}\right) \prod_{j=0}^{\beta(t)-1}\left(1+\tau_{j} F_{j}\left(T_{j-1}\right)\right)
$$


Le numéraire $B_{d}(t)$ s'interprète comme la valeur actuelle d'un investissement consistant à partir d'une unité monétaire et à investir à chaque échéance dans des obligations zéro-coupons pour le prochain ténor ${ }^{5}$ (l'indice "d" dans $B_{d}$ fait référence à l'écriture discrète de la valeur de l'investissement).

La mesure $Q_{d}$ associée au numéraire $B_{d}$ est appelé la "mesure spot».

Sous cette mesure, la dynamique du taux forward s'écrit:

$$
d F_{k}(t)=\sigma_{k}(t) F_{k}(t) \sum_{j=\beta(t)}^{k} \rho_{j, k} \frac{\tau_{j} \sigma_{j}(t) F_{j}(t)}{1+\tau_{j} F_{j}(t)} d t+\sigma_{k}(t) F_{k}(t) d Z_{k}^{d}(t)
$$

En utilisant la discrétisation d'Euler on peut écrire pour $i<k$ (Hill [2009] et Brigo et Mercurio [2007]):

$$
F_{k}\left(T_{i+1}\right)=F_{k}\left(T_{i}\right) \exp \left(\sigma_{k}\left(T_{i}\right)\left(\sum_{j=i+1}^{k} \rho_{j, k} \frac{\tau_{j} \sigma_{j}\left(T_{i}\right) F_{j}\left(T_{i}\right)}{1+\tau_{j} F_{j}\left(T_{i}\right)}-\frac{1}{2} \sigma_{k}\left(T_{i}\right)\right) \tau_{i}+\sigma_{k}\left(T_{i}\right) \sqrt{\tau_{i}} \varepsilon_{i}\right)
$$

La variable $\varepsilon_{i}$ suit une loi normale centrée réduite et $\left\langle\varepsilon_{i}, \varepsilon_{j}\right\rangle=\rho_{i, j}$.

Cette formule récurrente permet de diffuser les taux forward à chaque date en partant des taux forward observés à la date 0 . Ces derniers s'écrivent:

$$
F_{k}(t=0)=\frac{e^{L\left(0, T_{k}\right) T_{k}-L\left(0, T_{k-1}\right) T_{k-1}}-1}{\tau_{k}}
$$

\subsubsection{Prix d'une obligation zéro-coupon}

Le prix d'une obligation zéro-coupon s'écrit pour $j<k$ :

$$
P\left(T_{j}, T_{k}\right)=\prod_{i=j+1}^{K} \frac{1}{1+\tau_{i} F_{i}\left(T_{j}\right)}
$$

et $P\left(T_{j}, T_{j}\right)=1$.

La diffusion des taux forwards permet donc de diffuser le prix des obligations zéros coupon. 


\subsubsection{Modèles de volatilité instantanée et modèle de corrélation}

La valorisation d'obligations ou d'options nécessite la spécification de la forme de la volatilité instantanée et des corrélations.

Plusieurs modélisations de la volatilité instantanée des taux forward sont proposées dans la littérature (Brigo et Mercurio [2007] et Rebonato [2004]). Deux situations peuvent être distinguées:

- Les modèles à volatilité constante par morceau impliquant que pendant de courts intervalles de temps, la volatilité d'un taux forward est constante;

- Les modèles proposant l'utilisation d'une forme paramétrique impliquant que la volatilité est une fonction du temps, de la maturité et d'un ensemble de paramètres.

Dans le cadre de nos travaux, nous retenons le modèle de volatilité suivant présenté dans Rebonato [2004]:

$$
\sigma_{i}(t)=\left(a\left(T_{i-1}-t\right)+b\right) e^{-c\left(T_{i-1}-t\right)}+d
$$

Cette forme intégrant un terme linéaire avec une décroissance exponentielle caractérise la courbure de la dynamique de la volatilité et permet la formation d'une bosse. Cela implique également que la volatilité converge asymptotiquement vers $d$ quand $T_{i}$ tend positivement vers l'infini. La Figure 2 présente une interprétation des paramètres de la fonction de volatilité de Rebonato de la forme: $f(t)=(a t+b) e^{-c t}+d$ où $a=b=5 \%, c=20 \%$ et $d=16 \%$.

\section{FIGURE 2 Interprétation du modèle de volatilité de Rebonato}

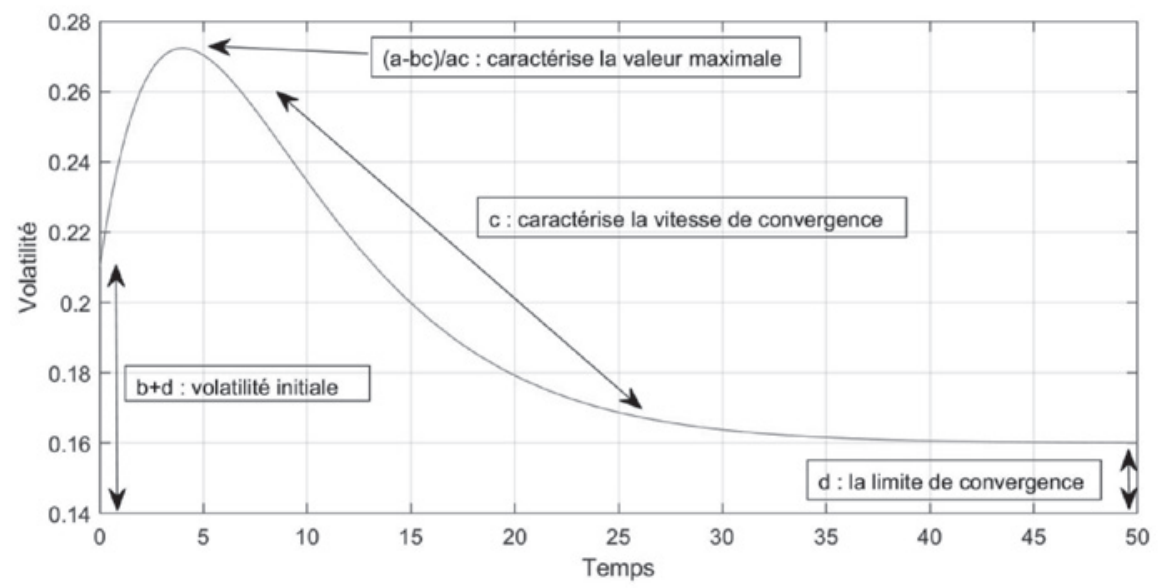


Pour le modèle de corrélations, nous avons retenu la forme paramétrique suivante présentée dans Rebonato [2004]:

$$
\rho_{i j}(t)=\exp \left(-\beta\left|T_{i}-T_{j}\right|\right)
$$

Cette forme paramétrique de la corrélation implique que plus les taux à terme sont éloignés, moins ils sont corrélés. De plus, pour tout $\beta$ positif, la matrice de corrélation générée est une matrice de corrélation admissible: c'est-à-dire une matrice réelle, symétrique avec des valeurs propres positives.

Cependant, cette forme est caractérisée par l'absence d'une dépendance temporelle: deux taux forward, séparés par la même distance temporelle $T_{i}-T_{j}$, auront la même corrélation, quelle que soit la date d'expiration $T_{i}$.

Le modèle de corrélation retenu peut être généralisé par la forme (Rebonato [2004] ):

$$
\rho_{i j}(t)=\exp \left(-\beta\left|g\left(T_{i}-t\right)-g\left(T_{j}-t\right)\right|\right)
$$

Ce modèle n'est pas retenu ici.

\subsubsection{Calibrage du modèle LMM sur des swaptions européennes}

Notons $S^{i, n}(t)$ le taux swap forward couvrant la période entre $T_{i}$ et $T_{n}$.

Le marché valorise les swaptions avec la formule de Black, ce qui suppose implicitement que le sous-jacent, le taux swap forward, est lognormal. Or cette hypothèse est incohérente avec celle supposant que le taux forward est log-normal.

En effet, la relation entre le taux swap forward $S^{i, n}(t)$, et les obligations zéro-coupon s'écrit:

$$
S^{i, n}(t)=\frac{P\left(t, T_{i}\right)-P\left(t, T_{n}\right)}{\sum_{j=i+1}^{n} \tau_{j} P\left(t, T_{j}\right)}
$$

Donc:

$$
S^{i, n}(t)=\sum_{j=i+1}^{n} w_{j}(\mathrm{t}) F_{j}(t)
$$

où : $w_{i}(t)=\frac{\tau_{i} P\left(t, T_{i}\right)}{\sum_{j=i+1}^{n} \tau_{j} P\left(t, T_{j}\right)}$ 
On note donc que si les taux forward sont log-normaux, le taux swap forward ne peut être log-normal.

Cette dernière expression est néanmoins importante car elle peut conduire à des approximations utiles. En effet, les taux swap forward peuvent être interprétés comme des moyennes pondérées des taux forward. Cependant, les poids " $w$ » dépendent des " $F$ ». Sur la base d'études empiriques montrant que la variabilité des " $w$ " est faible par rapport à la variabilité des " $F$ ", Brigo et Mercurio [2007] et Rebonato [1999], proposent d'approcher les " $w$ " par leurs valeurs initiales (déterministes) $w(0)$ et avoir donc:

$$
S^{i, n}(t)=\sum_{j=i+1}^{n} \mathrm{w}_{\mathrm{j}}(0) F_{j}(t)
$$

Supposons donc que le taux swap forward suit une dynamique log-normale. Sous une mesure de probabilité adaptée, le taux swap forward s'écrit:

$$
d S^{i, n}(t)=\sigma^{i, n}(t) S^{i, n}(t) d W_{t}^{i, n}
$$

Et soit $v_{i, n}^{2}$ la variance moyenne du taux swapforward sur l'intervalle $\left[0, T_{i}\right]$ multipliée par la longueur de l'intervalle. On a:

$$
v_{i, n}^{2}=\int_{0}^{T_{i}} \sigma^{i, n}(t)^{2} d t
$$

Le prix d'une swaption payeuse de strike $K$ s'écrit ( $D$ est le déflateur):

$$
D\left(0, T_{i}\right)\left(S^{i, n}\left(T_{i}\right)-K\right)^{+} \sum_{j=i+1}^{n} \tau_{j} P\left(T_{i}, T_{j}\right)
$$

Brigo et Mercurio [2007] montrent que le prix de la swaption payeuse formulé ci-dessus, coïncide avec celui donné par la formule de Black dont le paramètre de variance (multiplié par $T_{i}$ ) est $v_{i, n}^{2}$.

Par ailleurs la variance moyenne peut être approchée par la formule de Rebonato (cf. Rebonato [2004] et Brigo et Mercurio [2007]):

$$
v_{m, n}^{2}=\sum_{i, j=m+1}^{n} \frac{w_{i}(0) w_{j}(0) F_{i}(0) F_{j}(0) \rho_{i, j}}{S^{m, n}(0)^{2}} \int_{0}^{T_{m}} \sigma_{i}(t) \sigma_{j}(t) d t
$$


Minimiser une distance entre le prix du marché (donné par le modèle de Black) et le prix calculé par le modèle LMM, revient donc à minimiser la distance entre les volatilités des swaptions du marché (calculées donc par le modèle de Black) et les volatilités théoriques calculées par la formule de Rebonato.

Soit $\theta=(a, b, c, d, \beta)$ le vecteur de l'ensemble des paramètres du modèle (paramètres du modèle de volatilité et du modèle de corrélation). Le calibrage du modèle LMM sur les swaptions consiste à résoudre l'équation d'optimisation suivante ( $d$ est une distance):

$$
\hat{\theta}=\underset{\theta}{\operatorname{argmin}}\left(d\left(\left(\sigma_{m, n}^{\text {black }}\right)_{m, n},\left(v_{m, n}(\theta)\right)_{m, n}\right)\right)
$$

\subsubsection{Le modèle LMM et les taux négatifs}

Comme le modèle de Black, le modèle LMM est un modèle log-normal. L'utilisation de ce modèle dans l'état ne peut s'opérer dans un environnement économique caractérisé par des taux négatifs.

Afin de rendre le modèle LMM cohérent avec des taux négatif, un facteur de translation des taux forward peut être introduit. Ce facteur est appelé "shift» ou "displacement factor». On parle alors de modèle LMM décalé ou du displaced LMM model. Sous une mesure adaptée, la dynamique du modèle s'écrit en effet:

$$
d F_{k}(t)=\sigma_{k}^{(b)}(t)\left(F_{k}(t)+b\right) d Z_{k}(t) ; t \leq T_{k-1}
$$

Le facteur de translation est noté $h$.

Comme pour le modèle Black décalé une attention particulière doit être accordée au processus du choix du facteur de décalage. En effet la valeur du facteur de décalage devrait être suffisante pour éviter que les taux forward et le strike décalé ne passent en dessous de zéro; mais ne devrait pas être extrêmement élevée car dans ce cas le taux forward peut avoir des valeurs négatives significatives.

Les caractéristiques du modèle LMM décalé sont similaires au modèle LMM. Il peut être caractérisé par les mêmes modèles de volatilité et de corrélation présentés dans la section 2.1.4.4. Le vecteur de paramètre du modèle LMM décalé, $\theta^{(b)}=\left(a^{(b)}, b^{(b)}, c^{(b)}, d^{(b)}, \beta^{(b)}\right)$, dépend de la valeur du facteur de décalage choisi en amont. 
Une fois le modèle LMM décalé est calibré, le taux forward est calculé à chaque pas de temps par la formule suivante (Errais et al. [2004]):

$$
\begin{gathered}
F_{k}\left(T_{i+1}\right)=\left(F_{k}\left(T_{i}\right)+h\right) \exp \left(\sigma_{k}\left(T_{i}\right)\left(\sum_{j=i+1}^{k} \rho_{j, k} \frac{\tau_{j} \sigma_{j}\left(T_{i}\right)\left(F_{j}\left(T_{i}\right)+h\right)}{1+\tau_{j} F_{j}\left(T_{i}\right)}-\frac{1}{2} \sigma_{k}\left(T_{i}\right)\right) \tau_{i}\right. \\
\left.+\sigma_{k}\left(T_{i}\right) \sqrt{\tau_{i}} \varepsilon_{i}\right)-b
\end{gathered}
$$

Le calibrage du modèle LMM décalé est similaire au modèle LMM.

En effet, soit $\bar{S}^{i, n}(t)$ le taux swap forward décalé:

$$
\bar{S}^{i, n}(t)=S^{i, n}(t)+b=\sum_{j=i+1}^{n} \mathrm{w}_{\mathrm{j}}(0)\left(F_{j}(t)+b\right)
$$

Le taux swap forward décalé suit une dynamique log-normale. Sous une mesure de probabilité adaptée, le taux swap forward décalé s'écrit donc:

$$
d \bar{S}^{i, n}(t)=\sigma_{b}^{i, n}(t) \bar{S}^{i, n}(t) d W_{t}^{i, n}
$$

Et soit $\left(v_{i, n}^{(b)}\right)^{2}$ la variance moyenne du taux swap forward décalé sur l'intervalle $\left[0, T_{i}\right]$ multipliée par la longueur de l'intervalle. On a:

$$
\left(v_{i, n}^{(b)}\right)^{2}=\int_{0}^{T_{i}} \sigma_{b}^{i, n}(t)^{2} d t
$$

Le prix d'une swaption payeuse de strike $K$ s'écrit ( $D$ est le déflateur):

$$
D\left(0, T_{i}\right)\left(\bar{S}^{i, n}\left(T_{i}\right)-(K+b)\right)^{+} \sum_{j=i+1}^{n} \tau_{j} P\left(T_{i}, T_{j}\right)
$$

Le prix de la swaption payeuse formulé ci-dessus, coïncide avec celui donné par la formule de Black appliqué au modèle de Black décalé dont le paramètre de variance (multiplié par $T_{i}$ ) est $\left(v_{i, n}^{(b)}\right)^{2}$.

En suivant la même démonstration présentée dans Brigo et Mercurio [2007] page 282, on peut prouver que:

$$
\left(v_{m, n}^{(b)}\right)^{2}=\sum_{i, j=m+1}^{n} \frac{w_{i}(0) w_{j}(0)\left(F_{i}(0)+b\right)\left(F_{j}(0)+b\right) \rho_{i, j}}{\left(S^{m, n}(0)+h\right)^{2}} \int_{0}^{T_{m}} \sigma_{i}^{(b)}(t) \sigma_{j}^{(b)}(t) d t
$$


Minimiser une distance entre le prix du marché (donné par le modèle de Black décalé) et le prix calculé par le modèle LMM décalé, revient donc à minimiser la distance entre les volatilités des swaptions du marché (calculées donc par le modèle de Black décalé) et les volatilités théoriques décalées calculées par la formule de Rebonato ci-dessus.

Notons que le facteur de décalage utilisé pour extraire les volatilités de marché par le modèle de Black décalé doit être égal au facteur de décalage utilisé pour construire les volatilités du modèle LMM décalé.

Soit $\theta^{(b)}=\left(a^{(b)}, b^{(b)}, c^{(b)}, d^{(b)}, \beta^{(b)}\right)$ le vecteur de l'ensemble des paramètres du modèle (paramètres du modèle de volatilité et du modèle de corrélation). Le calibrage du modèle LMM décalé sur les swaptions consiste à résoudre l'équation d'optimisation suivante ( $d$ est une distance):

$$
\hat{\theta}=\underset{\theta}{\operatorname{argmin}}\left(d\left(\left(\sigma_{m, n}^{\text {black-shift }}\right)_{m, n},\left(v_{m, n}^{(s b i f t)}(\theta)\right)_{m, n}\right)\right)
$$

Le paramètre $\theta$, résultat de cette optimisation, dépend de la valeur du facteur de décalage.

\subsection{Modèle actions et immobilier}

\subsubsection{La dynamique du modèle Black \& Scholes}

Le modèle Black-Scholes est un modèle mathématique du marché pour décrire la dynamique d'un actif (ou d'un indice). Le modèle, dans sa formulation classique, suppose que le prix de l'actif financier est un processus stochastique en temps continu caractérisé par l'équation différentielle stochastique suivante sous la probabilité risque neutre:

$$
\frac{d S_{t}}{S_{t}}=r(t) d t+\sigma_{t} d W_{t}
$$

Avec:

- $S_{t}$ est le prix de l'actif;

- $r(t)$ est le taux court instantané;

- $\sigma_{t}$ est la volatilité;

- $W_{t}$ est un mouvement brownien géométrique. 
Le modèle de Black-Scholes à volatilité constante ne peut reproduire l'ensemble des prix des options observés sur le marché pour un sousjacent donné, car leur volatilité implicite varie en fonction du strike et de la maturité (cf. Tankov [2011] et Hull [2007]). Pour prendre en compte le smile du marché, une solution naturelle est de modéliser la volatilité comme une fonction déterministe du temps et de la valeur du sous-jacent.

Nous avons néanmoins retenu un modèle de Black-Scholes dont la volatilité est déterministe et fonction uniquement du temps.

Le taux instantané retenu est celui issu des modèles de taux présentés dans les sections précédentes et est donc stochastique. Cela permet d'assurer l'absence d'opportunité d'arbitrage du prix modélisé.

\subsubsection{Solution de l'équation différentielle}

L'application du lemme d'Itô à $\ln \left(S_{t}\right)$ permet de prouver que la solution générale de l'équation différentielle stochastique s'écrit (Tankov [2011] et Tankov [2015]):

$$
S_{t}=S_{0} \exp \left(\int_{0}^{t} r(u) d u-\int_{0}^{t} \frac{\sigma_{u}^{2}}{2} d u+\int_{0}^{t} \sigma_{u} d W_{u}\right)
$$

- $D(0, T)=\exp \left(-\int_{0}^{t} r(u) d u\right)$ représente le déflateur stochastique sans risque observé à la date 0 dont l'échéance est $T$;

- $\Sigma_{\mathrm{t}}=\sqrt{\frac{\int_{0}^{t} \sigma_{u}^{2} d u}{t}}$ représente la volatilité annualisée du logarithme népérien du prix du sous-jacent à l'instant $t$.

soit:

- $E=\left\{T_{0}=0, \ldots, T_{M}\right\}$ un ensemble de dates d'intérêt tel que $\sigma_{i}$ est constante entre $T_{i-1}$ et $T_{i}$;

- $\left\{\tau_{1}, \ldots, \tau_{M}\right\}$ un ensemble tel que $\tau_{i}$ est la différence entre $T_{i-1}$ et $T_{i}$;

- $R_{i}=\frac{\ln \left(\frac{P\left(0, T_{i}\right)}{P\left(0, T_{i-1}\right)}\right)}{\tau_{i}}$ le taux spot annuel pour la période entre $T_{i-1}$ et $T_{i}$. 
La forme discrètisée du prix de l'actif s'écrit:

$$
S_{T_{i+1}}=S_{T_{i}} \exp \left(\tau_{i+1} \mathbf{R}_{\mathrm{i}+1}-\tau_{i+1} \frac{\sigma_{i+1}^{2}}{2}+\sigma_{i+1} \int_{T_{i}}^{T_{i+1}} d W_{u}\right)
$$

Et on a également:

$$
S_{T_{k+1}}=S_{0} \exp \left(\sum_{i=1}^{k+1} \tau_{i} R_{i}-\sum_{i=1}^{k+1} \tau_{i} \frac{\sigma_{i}^{2}}{2}+\sqrt{\sum_{i=1}^{k+1} \tau_{i} \sigma_{i}^{2}} \varepsilon_{k+1}\right)
$$

où $\varepsilon_{i+1}$ est une variable gaussienne centrée réduite.

Notons :

- $\Lambda_{\mathrm{k}}=\sum_{i=1}^{k} \tau_{i} \mathrm{R}_{\mathrm{i}}$ : le taux d'actualisation total de la date 0 à $T_{k}$;

- $\Sigma_{\mathrm{T}_{\mathrm{k}}}=\sqrt{\frac{\sum_{i=1}^{k} \tau_{i} \sigma_{i}^{2}}{T_{k}}}:$ la volatilité annualisée du logarithme de $S_{T_{k}}$.

\subsubsection{Valorisation d'une option d'achat européenne}

Nous avons choisi de valoriser les options d'achat par une méthode Monte-Carlo. Ce choix s'explique par:

- La formule fermée de Black-Scholes n'est pas applicable directement quand les taux d'intérêt sont stochastiques. Une formule fermée existe néanmoins pour le modèle de Black-Scholes combiné au modèle de taux Hull et White (cf. Haastrecht [2010]) mais n'est pas retenue ici;

- Une volonté d'appliquer la même méthode de valorisation quel que soit le modèle de taux utilisé pour assurer la comparabilité des résultats.

Le prix d'un call à l'instant $T$ sur un actif ne versant pas de dividende s'écrit pour $\mathrm{N}$ trajectoires simulées:

$C\left(0, S_{0}, T, K, \Sigma_{T}\right)=E\left(D(0, T)\left(S_{T}-K\right)^{+}\right)=\frac{1}{N} \sum_{j=1}^{N} D^{(j)}(0, T)\left(S_{T}^{(j)}-K\right)^{+}$

Le calibrage de la dynamique Black-Scholes présentée ci-dessus sous la probabilité risque neutre consiste à estimer l'ensemble des $\left\{\sigma_{i}\right\}_{i}$ ce qui revient à estimer l'ensemble des volatilités implicites $\left\{\Sigma_{i}\right\}_{i}$. 
Le calibrage du modèle sur des calls consiste à résoudre l'équation d'optimisation suivante ( $d$ est une distance) pour chaque $T$ :

$$
\widehat{\Sigma_{T}}=\underset{\Sigma_{T}}{\operatorname{argmin}}\left(d\left(\text { Call }_{T}^{\text {Marché }}, C\left(0, S_{0}, T, K, \Sigma_{T}\right)\right)\right)
$$

Notons que si l'on retient un modèle à volatilité constante: $\sigma_{i}=\sigma$, le calibrage du modèle sur des calls peut se présenter comme la résolution de l'équation d'optimisation suivante ( $d$ est une distance):

$$
\sigma=\underset{\sigma}{\operatorname{argmin}}\left(\sum_{T} d\left(\text { Call }_{T}^{\text {Marché }}, C\left(0, S_{0}, T, K, \sigma\right)\right)\right)
$$

\subsubsection{Calibrage du modèle Black et Scholes sur un indice actions}

Notons que le prix d'un call de maturité $T_{k}$ sur un indice actions est une fonction bijective de la volatilité annualisée $\Sigma_{\mathrm{T}_{\mathrm{k}}}$. Ce dernier paramètre, appelé volatilité implicite, est calculé directement à partir des prix des calls observés sur le marché. L'ensemble des volatilités locales $\left\{\sigma_{i}\right\}_{i}$ s'en déduit ensuite par récurrence.

\subsubsection{Calibrage du modèle Black et Scholes sur un indice immobilier}

Les mouvements des prix de l'immobilier peuvent être assimilés à ceux des prix des actions (Koivu et al. [2005], Djehiche et al. [2005] et Laurent et al. [2016]). Cependant, le marché des dérivés sur des actifs immobiliers n'est pas aussi liquide et profond que celui des actions.

Nous avons retenu donc un modèle Black-Scholes à volatilité constante pour décrire la dynamique d'un investissement en immobilier. Cette volatilité est calibrée sur les prix historiques.

\subsection{Modélisation de la structure de dépendance}

La modélisation de la structure de dépendance d'un générateur de scénarios économiques est complexe que ce soit en probabilité risque neutre ou en probabilité historique. Une présentation plus détaillée de l'introduction de structures de dépendance multivariée et non-linéaire (copules) en probabilité historique est proposée dans Armel et al. [2011]. Laurent et al. [2016] et Haastrecht [2010] présentent par ailleurs le modèle BSHW ou une corrélation implicite peut être estimée entre le bruit gaussien du modèles Hull \& White et celui du modèle Black-Scholes. 
La problématique des corrélations implicites des actifs financiers a été traitée par plusieurs papiers de la littérature financière (par exemple: Da Fonseca et al. [2007], Driessen et al. [2005], Walker [2005]). Nous ne connaissons cependant pas d'articles traitant cette problématique dans le cadre d'un générateur de scénarios économiques multivariés sous la probabilité risque neutre.

L'objectif de cette section est de présenter la démarche que nous avons retenue pour introduire une structure de dépendance entre les dynamiques présentées ci-dessus.

Notons que tous les modèles que nous avons retenus sont normaux ou log-normaux. Nous pouvons donc nous placer dans un espace vectoriel gaussien ou la corrélation décrit complètement la structure de dépendance.

Pour rappel, trois générateurs de scénarios économiques sont construits :

- GSE 1 composé d'un modèle Hull et White pour le taux sans risque instantané, un modèle Black et Scholes pour le prix du portefeuille d'actions et un modèle Black et Scholes pour le prix d'un investissement en immobilier;

- GSE 2 composé d'un modèle G2++ pour le taux sans risque instantané, un modèle Black et Scholes pour le prix du portefeuille d'actions et un modèle Black et Scholes pour le prix d'un investissement en immobilier;

- GSE 3 composé d'un modèle LMM pour le taux forward, un modèle Black et Scholes pour le prix du portefeuille d'actions et un modèle Black et Scholes pour le prix d'un investissement en immobilier.

Trois matrices de corrélation sont donc à déterminer.

Si nous prenons le GSE 1:

- Le processus de taux court instantané n'est pas observable sur le marché et la chronique anticipée du mouvement brownien implicite au modèle n'est pas accessible;

- Le marché n'offre pas d'information sur le prix futur du sous-jacent action mais donne accès uniquement à une information sur sa variabilité (volatilité implicite). La chronique du mouvement brownien sous-jacent n'est pas accessible; 
- Les investissements en immobilier sont calibrés sur des données historiques et non pas sur des données anticipées.

La démarche consistant à (1) construire les observations des aléas browniens pour (2) déduire leurs corrélations (3) que l'on utilise ensuite pour les simulations, n'est pas adaptée à notre problématique.

L'estimation de la corrélation implicite en utilisant des produits dérivés (comme pour le modèle $\mathrm{BSHW}$ ) est difficile en pratique dans un cadre multivarié. En effet, il faudra trouver au moins un produit dérivé liquide dont les sous-jacents sont l'indice actions et l'investissement en immobiliers pour estimer leur corrélation.

Par ailleurs, dans un modèle brownien vectoriel, le passage d'une mesure de probabilité historique à une mesure de probabilité risque neutre ne modifie ni les volatilités ni les corrélations (cf. Caja et Planchet [2010]).

Notre démarche vise donc à injecter, dans les GSE risque-neutres proposés, les corrélations historiques observées entre les variables suivantes $^{6}$ :

- $R_{1}^{(t)}$ le taux sans risque sur un an observé à la date $t$ et $S_{1}^{(t)}=\ln \left(R_{1}^{(t)}\right)$;

- $\boldsymbol{R}_{2}^{(t)}=A_{1}^{(t)}-\boldsymbol{R}_{1}^{(t)}$ le taux de rendement logarithmique annuel observé à la date $t$ de l'historique de l'indice actions diminué du taux sans risque un an;

- $R_{3}^{(t)}=I_{1}^{(t)}-R_{1}^{(t)} \quad$ le taux de rendement logarithmique annuel observé à la date $t$ de l'historique de l'indice immobilier diminué du taux sans risque un an.

Deux matrices de corrélation sont calculées:

- $M_{1}=\operatorname{corrélation}\left(R_{1}^{(t)}, R_{2}^{(t)}, R_{3}^{(t)}\right)$ la matrice de corrélation utilisée pour les GSE 1 et 2 ;

- $M_{2}=\operatorname{corrélation}\left(S_{1}^{(t)}, R_{2}^{(t)}, R_{3}^{(t)}\right)$ la matrice de corrélation utilisée pour le GSE 3 .

Nous supposons que ces deux matrices ne dépendent pas du temps et ne dépendent pas des variables modélisées. 
Le processus de simulation des variables corrélées est le suivant:

1. Simulation des taux d'intérêt indépendamment des autres variables économiques;

2. À chaque pas de projection $t$, calcul de la distribution du bruit gaussien, centré et réduit, sous-jacent au taux forward un an (pour les GSE 1 et 2) ou de son logarithme (pour le GSE3);

3. Simulation de deux bruits gaussiens corrélés au bruit gaussien calculé dans l'étape 2 suivant la matrice de corrélation $M_{i}(\mathrm{i}=1,2$ ou 3) par la méthode de Cholesky;

4. Utilisation des deux bruits gaussiens dans les simulations des indices actions et immobilier.

Cette approche de simulation de l'indice actions et de l'indice immobilier conditionnellement aux trajectoires du taux forward un an permet de construire un modèle risque neutre dont la structure de dépendance est cohérente avec les corrélations historiques observées.

\section{RÉFÉRENCES}

[1] Armel K., Planchet F., Kamega A. [2011] «Quelle structure de dépendance pour un générateur de scénarios économiques en assurance?", Bulletin Français d'Actuariat, vol. 11, n²2.

[2] Armel K., Planchet F. [2018] "Comment construire un générateur de scénarios économiques risque neutre destiné à l'évaluation du best-estimate des contrats d'épargne?", Assurances et Gestion des Risques, à paraître.

[3] Ballotta L. [2004] «Alternative framework for the fair valuation of participating life insurance contracts", Cass Business School, Actuarial Research Paper $n^{\circ} 157$.

[4] Baldvinsdóttir E. K., Palmborg L. [2011] «On Constructing a Market Consistent Economic Scenario Generator", Handelsbanken Liv.

[5] Brigo D., Mercurio F. [2007] «Interest Rate Models - Theory and Practice». $2^{\text {nd }}$ Edition. Springer.

[6] Caja A., Planchet F. [2010] "La mesure du prix de marché du risque: quels outils pour une utilisation dans les modèles en assurance?", Assurances et gestion des risques, Vol.78 (3-4). 
[7] Cheng P.K., Planchet F. [2018] «Stochastic Deflator for an Economic Scenario Generator with Five Factors ", Document de travail, ISFA

[8] Da Fonseca J., Grasselli M., Tebaldi C. [2007] "Option pricing when correlations are stochastic: an analytical framework ", Review of Derivatives Research, Volume 10, No 2, Mai (2007).

[9] Dastarac H., Sauveplane P. [2010] «Les déflateurs stochastiques: quelle utilisation en assurance?", Mémoire d'actuaire, ENSAE.

[10] Djehiche B., Hörfelt P. [2005] «Standard approaches to asset \& liability risk", Supported by the Swedish Insurance Federation. Scandinavian Actuarial Journal. 2005(5): 377-400.

[11] Driessen J., Maenhout P., Vilkov G. [2005] «Option-Implied Correlations and the Price of Correlation Risk", Advanced Risk \& Portfolio Management Paper.

[12] Errais E., Mauri G., Mercurio F. [2004] «Capturing the Skew in Interest Rate Derivatives: A Shifted Lognormal LIBOR Model with Uncertain Parameters", Working paper.

[13] Haastrecht A. [2010] «Pricing Long-term Options with Stochastic Volatility and Stochastic Interest Rates", Phd Thesis, Published by Wohrmann Print Service, Zutphen, The Netherlands.

[14] Hill J. [2009] "A Note on Implementing LIBOR Market Model», The Numerical Algorithms Group Ltd, Wilkinson House, working paper.

[15] Hull J., White A. [1990] "Pricing interest rate derivative securities", Review of Financial Studies 3, 573-92.

[16] Hull J., [2007] "Options, futures et autres actifs dérivés", PEARSON EDUCATION, Edition 6.

[17] Hull J., White A. [1994] «Numerical Procedures for Implementing Term Structure Models I: Single-Factor Models». The Journal of Derivatives 2, 7-16.

[18] Koivu M., Pennanen T., Ranne A. [2005] «Modeling assets and liabilities of a Finnish pension insurance company: a VEqC approach ", Scandinavian Actuarial Journal, 2005(1): 46-76.

[19] Laurent J.P., Norberg R., Planchet F. (editors) [2016] «Modelling in life insurance - a management perspective ", EAA Series, Springer.

[20] Planchet F., Kamega A., Thérond P.E. [2009] «Scénarios économiques en assurance - Modélisation et simulation", Paris : Economica. 
[21] Rebonato R. [1999] «On the Pricing Implications of the Joint Lognormal Assumption for the Swaption and Cap Market ", The Journal of Computational Finance 2, 57-76.

[22] Rebonato R. [2004] "Volatility and correlation the perfect hedger and the fox", 2nd ed. Chichester: John Wiley and Sons Ltd.

[23] Tankov P. [2011] "Calibration de Modèles et Couverture de Produits Dérivés", Université Paris-Diderot (Paris VII).

[24] Tankov P. [2015] "Surface de volatilité», Université Paris-Diderot (Paris VII).

[25] Vasicek O. [1977] «An Equilibrium Characterization of the Term Structure", Journal of Financial Economics 5, 177-188.

[26] Walker M.B. [2005] «Risk-Neutral Correlations in the Pricing and Hedging of Basket Credit Derivatives ", Journal of Credit Risk.

\section{NOTES}

1. Kamal Armel est actuaire qualifié/certifié et fondateur d'ARMEL Consulting.

Contact: kamal.armel@armelconsulting.fr

2. Frédéric Planchet est Professeur à l'ISFA et actuaire associé à PRIM'ACT.

Contact: frederic@planchet.net

3. Se référer à Brigo et Mercurio [2007] pour une description détaillée de cette famille.

4. Le modèle SABR, ou modèle "Stochastic Alpha, Beta, Rho", est un modèle de volatilité stochastique généralisant le modèle log-normal de Black et permettant de valoriser les caps/floors et swaptions par des formules fermées de type Black.

5. Temps restant jusqu'à la maturité.
6. Pour une discrétisation annuelle, notons que: $\ln \left(\frac{S_{i+1}}{S_{i}}\right)-R_{i+1}=\frac{\sigma_{i+1}^{2}}{2}+\sigma_{i+1} N(0,1)$. Le rendement de l'indice diminué du taux sans risque est gaussien sous la probabilité risque neutre. 